\title{
DIVISION III / WORKING GROUP COMMITTEE ON SMALL BODIES NOMENCLATURE
} COMITÉ DE LA NOMENCLATURE DES PETITS CORPS CÉLESTES

\author{
CHAIR \\ SECRETARY \\ Div. III representative \\ MPC representative \\ CBAT representative \\ WG-PSN representative
}

MEMBERS

\author{
Jana Tichá \\ Brian G. Marsden \\ Edward L. G. Bowell (> 2006) \\ Iwan P. Williams (until 2006) \\ Brian G. Marsden \\ Daniel W. E. Green (for comet names only) \\ Kaare Aksnes (until 2006) \\ Rita M. Schulz (since 2006) \\ Michael F. A'Hearn, Julio A. Fernández, \\ Pamela Kilmartin, Yoshihide Kozai (until 2006), \\ Daniela Lazzaro, Syuichi Nakano (since 2006), \\ Keith S. Noll (since 2006), \\ Lutz D. Schmadel (editor DMPN), \\ Viktor A. Shor, Richard M. West (until 2006), \\ Gareth V. Williams, Donald K. Yeomans, \\ Jin Zhu
}

TRIENNIAL REPORT 2006-2009

\section{Comets}

A total of 701 comets received names between July 2005 and June 2008. Comets observed only from the SOHO and STEREO missions, as well as further comets recognized from the long-defunct SOLWIND satellite, accounted for 520 of these names.

\section{Minor planets}

Names for 2228 minor planets were approved and published in the Minor Planet Circulars in the period July 2005 - June 2008. In October 2005 the milestone of the 100 000th numbered minor planet was reached. There are now nearly 190000 numbered minor planets. As of June 2008, the total number of named minor planets was 14574 . This marked a reduction compared to the 2766 names of the previous triennium. This was part of conscious effort to limit the naming process.

In the resolution adopted at the IAU General Assembly in July 2003 and printed on the MPC 49221 the Committee on Small-Body Nomenclature (CSBN) recognized the need to limit the number of minor planets named. It requested individual discoverers and observing teams to propose no more than two names every two months (corresponding to the timescale generally adopted for the publication of new names in the MPC). The CSBN also set a practical limit of 100 name proposals for consideration in each bimonthly batch. The CSBN members think that it is far better to concentrate on having 
a smaller number of meaningful names with broad international appeal than to name all the tens of thousands of newly numbered main-belters. The majority - but not all of the discoverers cooperate, and this system seems to work. The CSBN continued to examine ways of limiting the naming process, particularly in the context of future years when the PanSTARRS and LSST projects become operational.

The CSBN reserves the right to accept additional names that are considered particularly meritorious, especially for traditional cases such as the those of planetary astronomers honored at the triennial Asteroids, Comets and Meteors meetings and of the science-fair students and mentors honored by LINEAR.

\section{Most significant names}

Minor planet (100000) Astronautica has been named to recognize the fiftieth anniversary of the start of the Space Age, inaugurated by the launching of the first artificial earth satellite on 4 October 1957. The name is associated with this significant number, as space is defined to begin at an altitude of 100000 meters above the earth's surface. The name was given on the MPC 60731 in September 2007.

The first names for a recently discovered dwarf planet and its satellite were adopted in September 2006. Following near-unanimous acceptance by both the CSBN and the Working Group on Planetary-System Nomenclature (in consultation with the discovery team), the IAU Executive Committee approved the names Eris for (136199) 2003 UB_313 and Dysnomia for its satellite (136199) Eris I. These names were published on IAUC 8747 and MPC 57800 .

Names for four smaller outer-solar system bodies and their satellites (or binary objects) were approved by the CSBN during this triennium. They are (42355) Typhon and (42355) Typhon I (Echidna), published on IAUC 8778 and MPC 57951; (65489) Ceto and (65489) Ceto I (Phorcys), published on IAUC 8778 and MPC 57952; (88611) Teharonhiawako and (88611) Teharonhiawako I (Sawiskera), published on IAUC 8840 and MPC 59388; and (66652) Borasisi and (66652) Borasisi I (Pabu), published on MPC 60731. The first two pairs are 'extended Centaurs' with mean distances beyond Neptune, and the other pairs are 'cubewanos' or 'classical Kuiper-Belt Objects'.

\section{Name for transneptunian dwarf planet category}

After introducing the category of dwarf planets, the IAU followed up on its footnote to Resolution 6 at the 2006 General Assembly and initiated a search for a name for transneptunian dwarf planets that are similar to Pluto. In June 2007 the CSBN received a request from the General Secretary of the IAU to propose such a name. After a long and wide discussion, at the end of August the CSBN voted on the various suggestions that had been proposed. These choices involved words related to the name of the prototype object Pluto, to the discoverer of Pluto, as well as to the transneptunian location and to the presumed physical physical nature of these objects. The name finally selected by the CSBN, plutoid, was approved by the IAU Executive Committee at its meeting in Oslo, Norway, in May 2008.

\section{Development of guidelines}

The CSBN has been working on the further development of guidelines for minor planet naming. 
A naming convention has been proposed for non-resonant objects with $a>30.07 \mathrm{AU}$ and 5.2 AU $<q<30.07 \mathrm{AU}$. These objects are in chaotic and short-lived orbits very similar to the orbits of the Centaurs. The proposed convention would name such objects for mythical creatures, particularly hybrids (human/animal, animal/animal) and shapeshifters. Lesser bodies in binary or multiple systems are to be named for closely related mythical characters such as siblings, spouses, parents and children, or other closely related characters. Examples are the Typhon-Echidna and Ceto-Phorcys pairs mentioned above.

Amplifying the guideline concerning commercial names, the CSBN proposed that sports-related names should not be for sports teams, but be limited to meritorious individuals in the world of sport.

\section{Miscellanous}

An addendum to the fifth edition of the Dictionary of Minor Planet Names was published by Springer-Verlag (Berlin-Heidelberg-New York) in 2006. Edited, as in the past, by L. D. Schmadel (Astronomisches Rechen-Institut, Heidelberg), it contains discovery and naming information on minor planets and their satellites that were named during 2003 -005, together with amendments to previously published citations.

Brian G. Marsden

secretary of the Commission 